\title{
लSTATE
}

\section{Age-Related Macular Degeneration (AMD)}

\section{What is AMD?}

AMD is a common eye disease. The macula is in the back of your eye in the center of the retina. It is the size of this capital " $\mathrm{O}$ " and is responsible for clear, sharp central or forward vision. You need it to see fine details for everyday tasks such as writing, driving, reading and sewing. AMD occurs when the macula is damaged or deteriorates in one or both eyes.

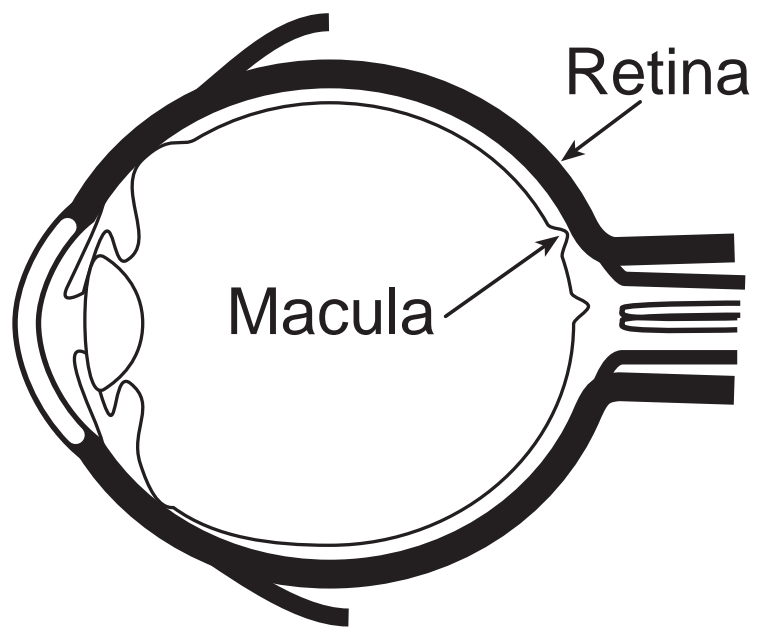

AMD is the leading cause of blindness in people over the age of 65 . An additional 1.7 million people suffer partial vision loss because of this disease. Vision lost to AMD is permanent.

As a person ages, the risk of developing this eye disease increases. Middle-aged people have a 2 percent risk of getting AMD. One out of 3 people over the age of 75 get AMD.

\section{Symptoms of AMD}

Early signs of AMD are the increased time it takes to adapt to light/dark environments, blurred vision, and increased sensitivity to glare. Straight lines may appear wavy. Colors may appear to be faded. The center of vision gradually becomes dark and empty.

\section{There are two types of AMD:}

"Dry" AMD - Ninety percent of people with AMD have this type. The light-sensing cells in the eye break down, and the tissue thins. Vision loss is gradual and painless.

"Wet" AMD - Ten percent of people with the "dry" type of AMD will progress to the more serious or "wet" form of this disease. New blood vessels develop in the macula in an effort to supply blood and nutrients to the thinning tissue and damaged light-sensing cells. These new blood vessels are fragile and often leak blood and fluid under the macula. This causes a greater and more rapid loss of central vision.

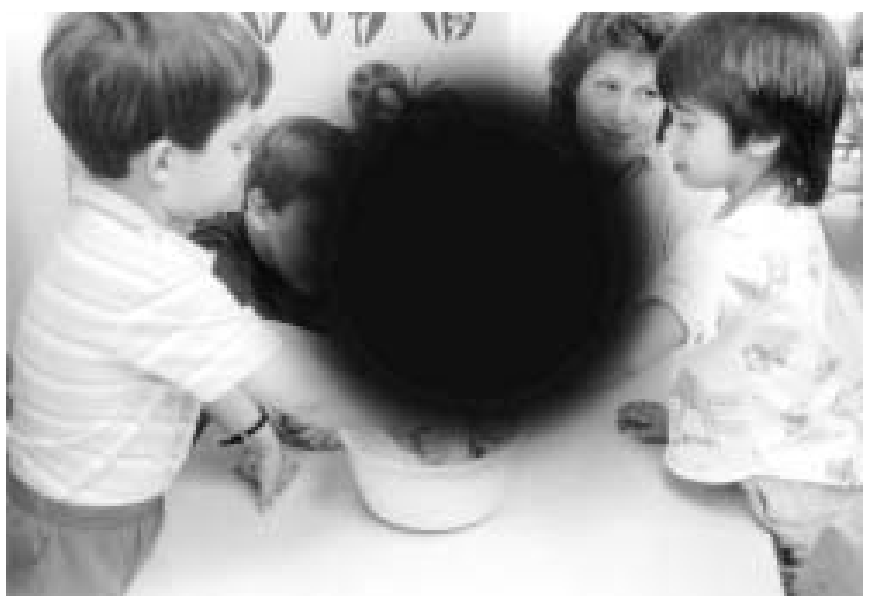

This is how someone with AMD might see. Central or forward vision is lost as the condition progresses. 


\section{Foods can help reduce your risk of developing age-related macular degeneration (AMD).}

\section{Eat lots of foods high in antioxidants:}

People who eat plenty of foods with antioxidants are at decreased risk of developing AMD. Antioxidants work to protect the retina from damaging molecules created by our bodies and from external factors. They may be able to prevent AMD or slow its progression.

Certain antioxidants, especially lutein and zeaxanthin, beta-carotene, vitamins $\mathrm{C}$ and $\mathrm{E}$, and zinc help keep your eyes healthy. Lutein and zeaxanthin form a yellow pigment in the macula of the eye. This pigment acts as a filter, protecting the eye from the sun's damaging rays. You can increase the amount of this pigment in your eyes by eating foods high in lutein and zeaxanthin.

Food sources of lutein: spinach, collard greens, kale, dark green leafy vegetables, egg yolk, corn, kiwi fruit, green peppers, peas, pumpkin, zucchini squash, butternut squash, yellow squash, red grapes, cucumber and celery.

Food sources of zeaxanthin: egg yolk, corn, red bell peppers, oranges, orange juice, mango, honeydew melon and seedless grapes.

Food sources of beta-carotene: red, deep orange, and yellow fruits and vegetables, and dark green leafy vegetables, such as sweet potatoes, broccoli, carrots and squash.

Food sources of vitamin $\mathbf{C}$ : citrus fruits and juices (including oranges, grapefruit, lemons, tangerines), strawberries, tomatoes and green peppers.

Food sources of vitamin E: seeds, nuts, vegetable oils, margarine and seafood.

Food sources of zinc: oysters, wheat germ, seeds, liver, spices and meats.

Eat a diet low in animal and tropical fats: Diets high in saturated fat and cholesterol can nearly double the risk of developing AMD.

Note: A balanced diet rich in fruits and vegetables can also offer protection from many other diseases, such as cancer, heart disease, stroke, and other eye problems.

Other ways to reduce the risk of developing AMD:

* Have annual eye examinations by a qualified eye care professional. AMD can be diagnosed before it causes blindness.

* Protect your eyes from sunlight exposure. Wear a brimmed hat and sunglasses to protect your eyes from UV-light and reduce sun damage to the macula.

* Don't smoke. Smoking can increase your risk for AMD, especially "wet" AMD.

* Control high blood pressure. People with uncontrolled high blood pressure have increased risk of developing AMD.

* Post-menopausal women, replace hormone levels. Post-menopausal women who are not on hormone replacement therapy or estrogen therapy may have increased risk of developing AMD.

\section{* Get regular physical activity.}

Susan Boger, Assistant Professor, Department of Human Nutrition,

and Mary L. Meck Higgins, Extension Specialist, Department of Human Nutrition, Kansas State University.

Reviewed by Carol Ann Holcomb, Associate Professor, Department of Human Nutrition, Kansas State University.

The information in this fact sheet is for educational purposes only and is not meant as a substitute for advice from your health care provider.

Publications from Kansas State University are available on the World Wide Web at: http://www.oznet.ksu.edu

Contents of this publication may be freely reproduced for educational purposes. All other rights reserved. In each case, credit Susan Boger and Mary L. Meck Higgins, “Age-Related Macular Degeneration (AMD),” Kansas State University, August 1999.

Kansas State University Agricultural Experiment Station and Cooperative Extension Service

It is the policy of Kansas State University Agricultural Experiment Station and Cooperative Extension Service that all persons shall have equal opportunity and access to its educational programs, services, activities, and materials without regard to race, color, religion, national origin, sex, age or disability. Kansas State University is an equal opportunity organization. Issued in furtherance of Cooperative Extension Work, Acts of May 8 and June 30, 1914, as amended. Kansas State University, County Extension Councils, Extension Districts, and United States Department of Agriculture Cooperating, Marc A. Johnson, Director.

File code: Human Nutrition 9-1 